\title{
Incidence and correlation of metabolic syndrome and kidney stones in a healthy screening population
}

\author{
Feng Qiu ${ }^{1 \#}$, Yunfeng $\mathrm{Xu}^{2 \#}$, Xiaodong $\mathrm{Ji}^{3 \#}$, Jinxian $\mathrm{Pu}^{1}$, Jian Zhou ${ }^{4}$, Yuhua Huang ${ }^{1}$ \\ ${ }^{1}$ Department of Urology, the First Affiliated Hospital of Soochow University, Suzhou, China; ${ }^{2}$ Department of Urology, KunShan Second People's \\ Hospital, Suzhou, China; ${ }^{3}$ Department of Health Management Center, the First Affiliated Hospital of Soochow University, Suzhou, China; ${ }^{4}$ Center \\ for Reproductive Medicine, Dushuhu Public Hospital Affiliated to Soochow University, Suzhou, China \\ Contributions: (I) Conception and design: F Qiu, J Pu; (II) Administrative support: Y Huang; (III) Provision of study materials or patients: X Ji; (IV) \\ Collection and assembly of data: Y Xu, J Zhou; (V) Data analysis and interpretation: F Qiu, J Zhou; (VI) Manuscript writing: All authors; (VII) Final \\ approval of manuscript: All authors. \\ "These authors contributed equally to this work. \\ Correspondence to: Jinxian Pu. Department of Urology, the First Affiliated Hospital of Soochow University, Suzhou 215006 , China. \\ Email: pjx62@sina.com; Jian Zhou. Center for Reproductive Medicine, Dushuhu Public Hospital Affiliated to Soochow University, Suzhou 215008, \\ China. Email: drchouj@163.com.
}

Background: To study the incidence of metabolic syndrome (MetS) and kidney stones in a healthy screening population and to explore the correlation between them.

Methods: The physical examination data of 11,827 people screened at the First Affiliated Hospital of Soochow University from August 2019 to July 2020 were analyzed. MetS diagnostic criteria were based on the 2004 guidelines of Chinese Diabetes Society (CDS). Multivariate logistic regression was used to analyze the correlation between MetS and various characteristics and kidney stones. Trend analysis was represented by $\mathrm{P}$ value, and $\mathrm{P}<0.05$ indicated statistical significance.

Results: The present study comprised 6,570 males (55.6\%, aged 46.15 \pm 13.653 years) and 5,257 females (44.4\%, aged $41.41 \pm 11.712$ years). Of these, 1,036 (8.8\%) had kidney stones and 1,552 (13.1\%) had MetS. Among the MetS patients, 35.1\% had a body mass index (BMI) $\geq 25,27.7 \%$ had hypertension, 10.8\% had hyperglycemia, and 31.2\% had dyslipidemia. Kidney stone morbidity was $14.5 \%$ in the MetS group and $7.9 \%$ in the non-MetS group $(\mathrm{P}<0.05)$. As the number of MetS characteristics increased, kidney stone morbidity showed a linear increasing trend $(\mathrm{P}<0.05$ for trend). With an increase in BMI and blood triglycerides (TG), and a decrease in lipoprotein cholesterol (HDL-C), the incidence of kidney stones had an increasing trend $(\mathrm{P}<0.05$ for trend). Sex, age and MetS were independent risk factors for the occurrence of kidney stones, with and odds ratio (OR) of 1.493 [95\% confidence interval (CI): 1.264-1.763] for MetS. Of the MetS characteristics, BMI $\geq 25$ and blood pressure (BP) $\geq 140 / 90 \mathrm{mmHg}$ were independent risk factors for kidney stones, with OR values of 1.209 (95\% CI: 1.047-1.396) and 1.248 (95\% CI: 1.071-1.453), respectively.

Conclusions: MetS is an independent risk factor for kidney stones. Appropriate medication and dietary advice may help to correct urinary metabolic abnormalities and prevent the recurrence of kidney stones.

Keywords: Metabolic syndrome (MetS); kidney stones; obesity; hypertension

Submitted Jun 29, 2021. Accepted for publication Sep 15, 2021.

doi: $10.21037 /$ tau-21-689

View this article at: https://dx.doi.org/10.21037/tau-21-689 


\section{Introduction}

Metabolic syndrome (MetS) is associated with several metabolic disorders, including obesity (mainly abdominal obesity), fasting, postprandial hyperglycemia, hypertension, and dyslipidemia (1). These conditions are linked to a common mechanism: insulin resistance (2). Although there are several definitions of MetS, such as the World Health Organization (WHO 1999), the National Cholesterol Education Program-Third Adult Treatment Panel (NCEPATPIII 2005), the International Diabetes Federation (IDF 2005) (3). We choosed the guidelines of the Chinese Diabetes Society (CDS 2004), Diagnostic criteria for MetS was listed in the Methods. MetS has become a major health challenge globally, as it is associated with an increased risk of cardiovascular disease and type 2 diabetes (4). MetS morbidity varies by age, sex, race, and the definition used. MetS is associated with many urinary disorders, such as erectile dysfunction, benign prostatic hyperplasia, prostate cancer, stress urinary incontinence, chronic kidney disease, and kidney stones (5). The recurrence rate of kidney stones is high, with a $50 \%$ chance of recurrence within 7 years without causal factor analysis and appropriate intervention (6). For patients treated with percutaneous nephrolithotomy (PCNL), the recurrence rate of kidney stones during long-term follow up is $>40 \%$, and the risk of postoperative kidney stone recurrence in MetS has been found to be 3.2 times higher when compared with a control group (7). MetS has been found to be associated with renal function deterioration during long-term follow up (7). Although treatments for removing kidney stones have improved significantly over the past few decades, optimizing preventive measures to reduce the risk of kidney stone recurrence remains a major issue (8). Kidney stones may be a systemic disease that represents the interaction of multiple metabolic risk factors, and many researchers support interventional studies on the impact of MetS on the risk of kidney stone development (9). Therefore, in the present study, we analyzed the incidence of MetS and kidney stones in a healthy screening population and explored the correlation between them. This is the first cross-sectional study of a large sample in the Chinese population, and the metabolic syndrome standard developed by the Diabetes Society of Chinese Medical Association is more closely related to the characteristics of Chinese people. In addition, the statistical method of $\mathrm{P}$ for trend is introduced in this study to facilitate the trend test. We present the following article in accordance with the STROBE reporting checklist (available at https://dx.doi.org/10.21037/tau-21-689).

\section{Methods}

\section{General data}

Data of 11,827 people who participated in a physical examination at the Physical Examination Center of the First Affiliated Hospital of Soochow University from August 2019 to July 2020 were included in the cross-sectional study. Urological system ultrasound examination and/or abdominal computed tomography were performed for all patients to determine the presence of kidney stones. Past medical history and BMI, BP, and blood biochemical and urine routine examination results were collected for all participants.

\section{Diagnostic criteria for MetS}

MetS diagnosis was based on the 2004 guidelines of the Chinese Diabetes Society. MetS can be diagnosed with three or all of the following four components: (I) Overweight or obesity: $\mathrm{BMI} \geq 25\left(\mathrm{~kg} / \mathrm{m}^{2}\right)$; (II) hyperglycemia: fasting blood glucose $\geq 6.1 \mathrm{mmol} / \mathrm{L}$, and/or patients diagnosed with and treated for diabetes; (III) hypertension: systolic blood pressure (SBP)/diastolic blood pressure (DBP) $\geq 140 / 90 \mathrm{mmHg}$, and/or patients diagnosed and treated for hypertension; and (IV) dyslipidemia: $\mathrm{TG} \geq 1.7 \mathrm{mmol} / \mathrm{L}$ and (or) HDL-C $<0.9 \mathrm{mmol} / \mathrm{L}$ (males) or $<1 \mathrm{mmol} / \mathrm{L}$ (females).

\section{Statistical methods}

SPSS version 25.0 (SPSS Inc., Chicago, IL, United States) was used to process data. Single sample KolmogorovSmirnov test and QQ chart were used to judge the distribution of all measurement data. Mean \pm standard deviation was used for those conforming to normal distribution, and Median (Q1, Q3) was used for skewed distribution data. The $t$-test was used for the comparison between 2 groups of normal distribution data, and 1-way analysis of variance and polynomial linear comparison were used for the comparison between multiple groups of data. Mann-Whitney U-test was used for comparison of skewed distribution data between 2 groups, Kruskal-Wallis $\mathrm{H}$-test was used for the comparison of multiple groups, and Jonckheere-Terpstra test was used for the trend test. Enumeration data were expressed by frequency (percentage), and $\chi^{2}$-test was used for the comparison between groups. Univariate logistic regression was used to screen out 
statistically significant variables, and these variables were included in the multivariate logistic regression to analyze the correlation between MetS and each component (characteristic) and kidney stones. Trend analysis was represented by $\mathrm{P}$ value, and $\mathrm{P}<0.05$ indicated statistical significance.

\section{Ethical statement}

The study was approved by the Ethics Committee of First Affiliated Hospital of Soochow University (No. 178, 2021) and written informed consent was obtained from all patients. All procedures performed in this study involving human participants were in accordance with the Declaration of Helsinki (as revised in 2013).

\section{Results}

\section{General data}

The present study comprised 6,570 males (55.6\%, aged $46.15 \pm 13.653$ years) and 5,257 females $(44.4 \%$, aged $41.41 \pm 11.712$ years). Of these, 1,036 patients $(8.8 \%)$ had kidney stones and 1,552 patients (13.1\%) had MetS. Among all MetS patients, $35.1 \%$ had a BMI $\geq 25,27.7 \%$ had hypertension, $10.8 \%$ had hyperglycemia, and $31.2 \%$ had dyslipidemia (8.5\% low HDL-C, $28.5 \%$ high TG). The proportion with $\mathrm{BMI} \geq 25$ was the highest.

\section{Comparison of clinical indicators between the MetS group and non-MetS group}

As shown in Table 1, there were statistical differences between the 2 groups in age, sex, BMI, SBP, DBP, blood glucose, TG, HDL-C, urine $\mathrm{pH}$, urine specific gravity, urine white blood cell count, urine calcium oxalate crystal, blood urea nitrogen, blood creatinine, and blood uric acid. Age, male proportion, BMI, SBP, DBP, blood glucose, TG, urine specific gravity, positive rate of calcium oxalate crystal, blood urea nitrogen, blood creatinine, and blood uric acid in the MetS group were all higher than those in the nonMetS group, while the positive rate of HDL-C, urine $\mathrm{pH}$, and urine white blood cells were all lower than those in the non-MetS group.

Patients were grouped according to the number of MetS components (characteristics). Component 0 had 5,044 cases (42.6\%), component 1 had 3,004 cases (25.4\%), component 2 had 2,227 cases (18.8\%), component 3 had 1,250 cases
(10.6\%), and component 4 had 302 cases (2.6\%). Table 2 shows the statistical differences in all clinical indicators in the subgroup analysis $(\mathrm{P}<0.05)$. With an increase in the component score, age, male ratio, BMI, SBP, DBP, blood glucose, TG, urine specific gravity, positive rate of calcium oxalate crystal, blood urea nitrogen, blood creatinine, and blood uric acid gradually increased, and there was a linear increasing trend $(\mathrm{P}<0.05$ for trend). However, with an increase in the component score, HDL-C, urine $\mathrm{pH}$, positive rate of urine white blood cell, and positive rate of bacteria gradually decreased, and there was a linear decreasing trend $(\mathrm{P}<0.05$ for trend).

\section{Comparison of kidney stone morbidity between the MetS and non-MetS groups}

As shown in Table 3, kidney stone morbidity in the MetS group was $14.5 \%$ and $7.9 \%$ in the non-MetS group $(\mathrm{P}<0.05)$. In addition, we also observed that kidney stone morbidity had a linear increasing trend with an increase in scores of the MetS group ( $\mathrm{P}<0.05$ for trend).

\section{Correlation between MetS and kidney stone occurrence}

We converted metabolic syndrome into grade variables according to its characteristic numbers and calculated the Sperman correlation coefficient with the kidney stone occurrence, $r_{s}=0.107, \mathrm{P}<0.001$. Multivariate logistic regression analysis showed that sex, age, and MetS condition were independent risk factors for the occurrence of kidney stones (Table 4). The odds ratio (OR) of MetS was 1.493 [ $95 \%$ confidence interval (CI): $1.264-1.763$ ]. The highest risk of kidney stones was in the $\geq 33$ and $<43$-yearold subgroups. The risk in the $\geq 33$ and $<43$-year-old subgroups was 1.925 -fold (95\% CI: 1.556-2.383) compared with the $<33$-year-old subgroup. As shown in Table 5, compared with the 0 -component group, the risk of kidney stones in the 2-component group was 1.458-fold (95\% CI: 1.204-1.766), the risk of kidney stones in the 3-component group was 1.828 -fold (95\% CI 1.473-2.268), and the risk of kidney stones in the 4-component group was 1.773 times (95\% CI: 1.237-2.542). When converting MetS component scores into grade variables, $\mathrm{P}<0.05$ for trend indicated that there was a linear trend between MetS scores and kidney stones, and the incidence of kidney stones increased with the increase of group scores. As shown in Table 6, according to MetS (CDS 2004) standards, BMI, blood pressure, fasting blood glucose, TG, and HDL-C in the physical 
Table 1 Comparison of clinical indicators between the metabolic syndrome (MetS) group and the non-MetS group

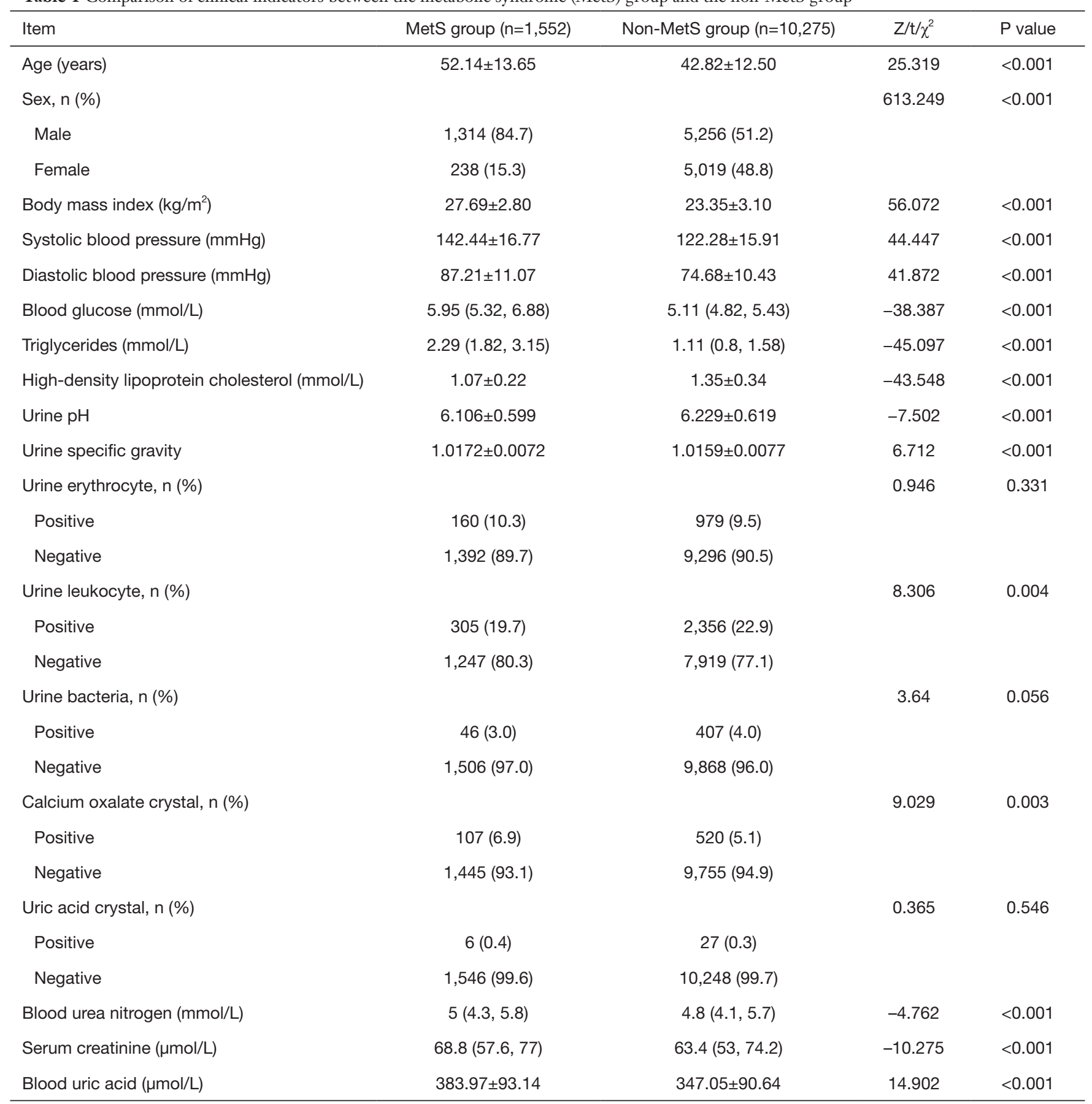

examination population were set as the dichotomous variables and were included in the model. The results indicated that $\mathrm{BMI} \geq 25$ and blood pressure $\geq 140 / 90 \mathrm{mmHg}$ were independent risk factors for kidney stones, with OR values of 1.209 (95\% CI: $1.047-1.396)$ and 1.248 (95\% CI: 1.071-1.453), respectively. As shown in Table 7, BMI, SBP,
DBP, fasting blood glucose, TG, and HDL-C values in the physical examination population were grouped into the model by quartile spacing. The findings indicated that there was a linear relationship between BMI, TG, and HDL-C values and stones. With an increase in BMI, and TG, and a decrease in HDL-C, the incidence of kidney stones had an 
Table 2 Comparison of clinical indicators among different component (characteristic) numbers of metabolic syndrome

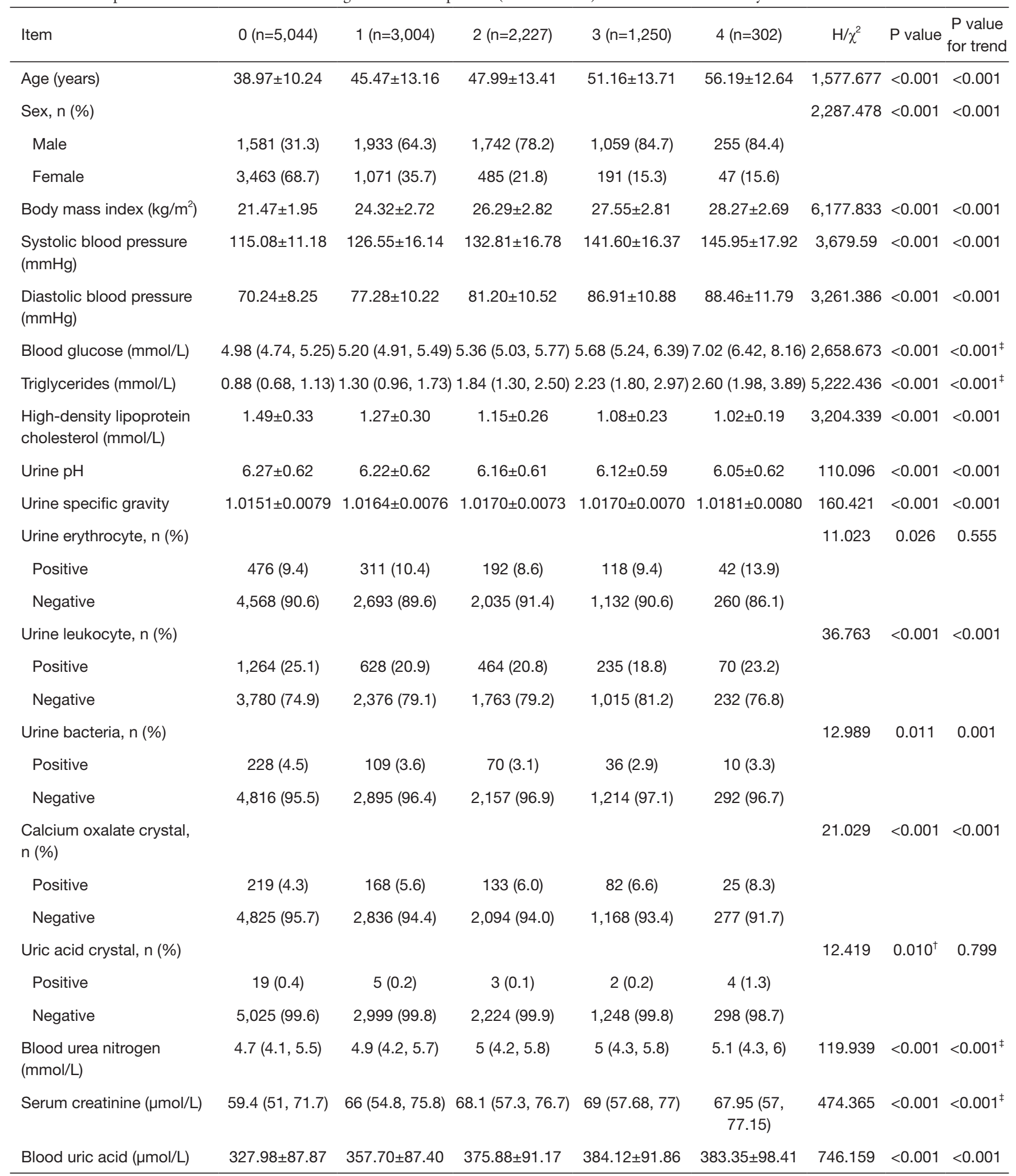

\footnotetext{
${ }^{\dagger}$, Fisher's exact test; ${ }^{\ddagger}$, Jonckheere-Terpstra test.
} 
Table 3 Comparison of the kidney stone morbidity between the metabolic syndrome (MetS) and non-MetS groups and between different component (characteristic) number groups

\begin{tabular}{|c|c|c|c|c|c|c|c|c|c|}
\hline Variable & \multicolumn{3}{|c|}{ MetS } & \multicolumn{5}{|c|}{ MetS component no. } & $\begin{array}{l}\mathrm{P} \text { value } \\
\text { for treno }\end{array}$ \\
\hline $\mathrm{n}$ & 10,275 & 1,552 & & 5,044 & 3,004 & 2,227 & 1,250 & 302 & \\
\hline Kidney stones (\%) & 7.9 & 14.5 & $<0.001$ & 5.8 & 8.7 & 11.5 & 14.6 & 14.2 & $<0.001$ \\
\hline Yes, $n(\%)^{\dagger}$ & $811(78.3)$ & $225(21.7)$ & & $294(28.4)$ & $261(25.2)$ & $256(24.7)$ & $182(17.6)$ & $43(4.1)$ & \\
\hline
\end{tabular}

${ }^{\dagger}, 1,036$ patients with kidney stones (100\%); ${ }^{\ddagger}, 10,791$ patients without kidney stones $(100 \%)$.

Table 4 Logistic regression analysis of risk factors for kidney stone formation

\begin{tabular}{|c|c|c|c|c|}
\hline Item & Crude OR (95\% Cl) & $P$ value & Adjusted OR $(95 \% \mathrm{Cl})$ & $P$ value \\
\hline Age (years) & & $<0.001$ & & $<0.001$ \\
\hline$<33$ & 1.000 & & 1.000 & \\
\hline $33-43$ & 2.061 (1.669-2.546) & $<0.001$ & $1.975(1.597-2.442)$ & $<0.001$ \\
\hline$\geq 53$ & $2.218(1.789-2.751)$ & $<0.001$ & $1.715(1.376-2.139)$ & $<0.001$ \\
\hline MetS condition & 1.979 (1.689-2.318) & $<0.001$ & $1.493(1.264-1.763)$ & $<0.001$ \\
\hline
\end{tabular}

$\mathrm{Cl}$, confidence interval; OR, odds ratio; MetS, metabolic syndrome.

Table 5 Logistic regression analysis of different MetS component (characteristic) number groups in patients with and without kidney stones

\begin{tabular}{|c|c|c|c|c|c|}
\hline Item & Crude OR $(95 \% \mathrm{Cl})$ & $P$ value & Adjusted $\mathrm{OR}^{\dagger}(95 \% \mathrm{Cl})$ & $P$ value & $P$ value for trend \\
\hline 0 component & 1.000 & & 1.000 & & \\
\hline 1 component & $1.537(1.293-1.828)$ & $<0.001$ & $1.180(0.983-1.417)$ & 0.076 & \\
\hline 2 components & $2.098(1.761-2.501)$ & $<0.001$ & $1.458(1.204-1.766)$ & $<0.001$ & \\
\hline 4 components & $2.682(1.902-3.782)$ & $<0.001$ & $1.773(1.237-2.542)$ & 0.002 & \\
\hline
\end{tabular}

${ }^{\dagger}$, confounders were adjusted for age and sex. $\mathrm{Cl}$, confidence interval; OR, odds ratio; MetS, metabolic syndrome.

increasing trend.

\section{Discussion}

The present cross-sectional study showed that kidney stone morbidity was $8.8 \%$ and MetS morbidity was $13.1 \%$ in the physical examination population. According to Jeong et al.'s study on a physical examination population in South Korea, kidney stone morbidity was $5.2 \%$ and MetS morbidity was
$13.7 \%$. These findings indicated that the morbidity of the 2 diseases is similar among East Asians (9).

$\mathrm{BMI}$ is considered a reliable alternative to waist circumference because they are highly correlated (10). In a number of previously published studies, BMI has been used to define obesity instead of waist circumference, there are many diagnostic criteria for MetS in China and globally (3). In the present study, BMI was used instead of waist circumference because the waist circumference 
Table 6 Logistic regression analysis of MetS-related clinical indicators in patients with and without kidney stones

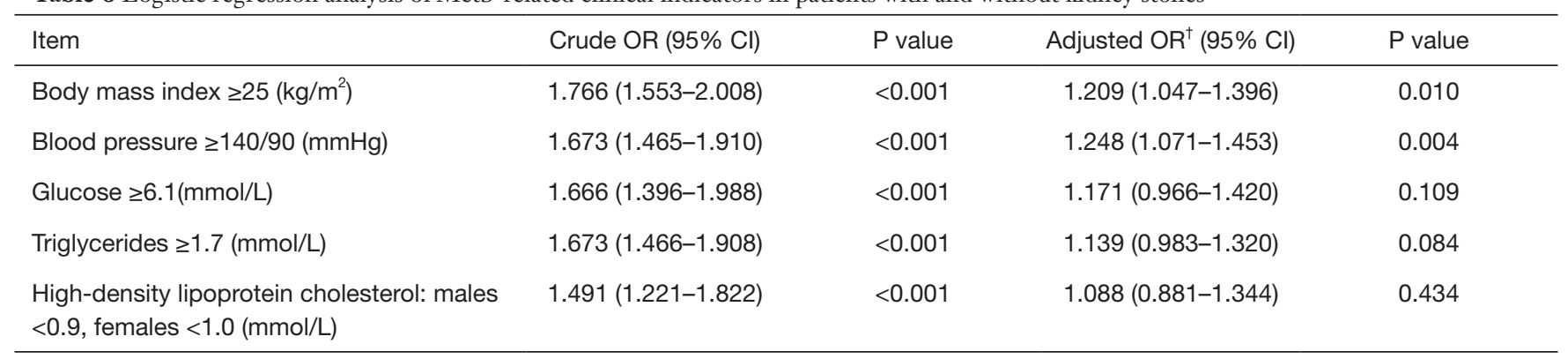

${ }^{\dagger}$, confounders were adjusted for age and sex. $\mathrm{Cl}$, confidence interval; OR, odds ratio; MetS, metabolic syndrome.

data of patients could not be collected. Taking race into account, CDS 2004 criteria were used to define overweight or obesity as $\mathrm{BMI} \geq 25$. Data in the present study showed that the proportion of MetS patients with a BMI $\geq 25$ was highest, suggesting that abnormal weight was the primary feature of MetS in Chinese population.

The urine $\mathrm{pH}$ of the MetS group was lower than that of the non-MetS group, and the higher the component score, the lower the $\mathrm{pH}$. Although the exact pathophysiological mechanism of the association between MetS and kidney stones is unknown, MetS is associated with changes in urinary composition, including decreased urinary $\mathrm{pH}$, reduced citrate excretion, and increased uric acid and calcium excretion, leading to an increased risk of kidney stone formation (9). Studies have shown that acid urine, caused by insulin resistance, is the cause of uric acid stone formation (11). Urinary $\mathrm{pH}$ affects the composition of stone formation. Unlike acid urine, alkaline urine $\mathrm{pH}$ promotes calcium phosphate stone formation (12).

The urine specific gravity of the MetS group was higher than that of the non-MetS group, and urine specific gravity showed an increasing trend with an increase in component number. Urine specific gravity is an important indicator reflecting water content of the body. Few previous studies have focused on the relationship between water intake and MetS. A study by NHANES (National Health and Nutrition Examination Survey), which included data from 3961 adults, showed that the highest urine specific gravity group had a higher MetS risk (OR: 1.6, 99\% CI: 1.0-2.7, $\mathrm{P}=0.01$ ) and diabetes risk (OR: 1.8, 99\% CI: 1.0-3.4, $\mathrm{P}<0.05)$ compared to the lowest quartile (13).

The positive rate of calcium oxalate crystals in the urine of the MetS group was higher than that of the non-MetS group. Further analysis based on the number of features also showed that the positive rate increased with an increase in the number of features. Sakhaee et al. indicated that the risk of $\mathrm{CaOx}$ (calcium oxalate) stone formation in patients without kidney stones increased with an increase in the number of MetS characteristics. However, the propensity to precipitate $\mathrm{CaOx}$ crystals was much higher in patients with kidney stones, but was not independently associated with an increase in the number of MetS features (14). The correlation between MetS and uric acid-based stones has been reported in previous literature. Serum uric acid was $383.97 \pm 93.14 \mu \mathrm{mol} / \mathrm{L}$ in the metabolic syndrome group and $347.05 \pm 90.64 \mu \mathrm{mol} / \mathrm{L}$ in the non-metabolic syndrome group, respectively, $\mathrm{P}<0.001$. The incidence of hyperuricemia in the metabolic syndrome group and the non-metabolic syndrome group was $33.3 \%$ and $19.1 \%$, respectively, $\mathrm{P}<0.001$. According to metabolic syndrome characteristics, the incidence of hyperuricemia from components 0 to 4 was $13.9 \%, 21.3 \%, 28.1 \%, 33.0 \%$ and $32.8 \%$, respectively. Similar to the research results of Cho et al. (15), our data showed that the blood uric acid value in MetS was significantly higher than that in the non-MetS group, and there was an increasing trend in blood uric acid with the increase in the number of features, but no difference was found in urine uric acid crystals between the 2 groups. The subgroup analysis showed that when there were 4 features, the positive rate of uric acid crystals was $13 \%$, which was higher than the other groups.

The indicators reflecting renal function, serum creatinine and urea nitrogen, in the MetS group were higher than those in the non-MetS group, and had an increasing trend with the increase in the number of features. Previous studies on the relationship between MetS and kidney function are limited. Kawamoto et al. showed that, for middle-aged and elderly Japanese, MetS was significantly associated with a decrease in eGFR (estimated glomerular filtration rate), while low HDL-C and increased HbA1c (glycosylated 
Table 7 Logistic regression analysis of MetS-related characteristics (quartile) in patients with and without kidney stones

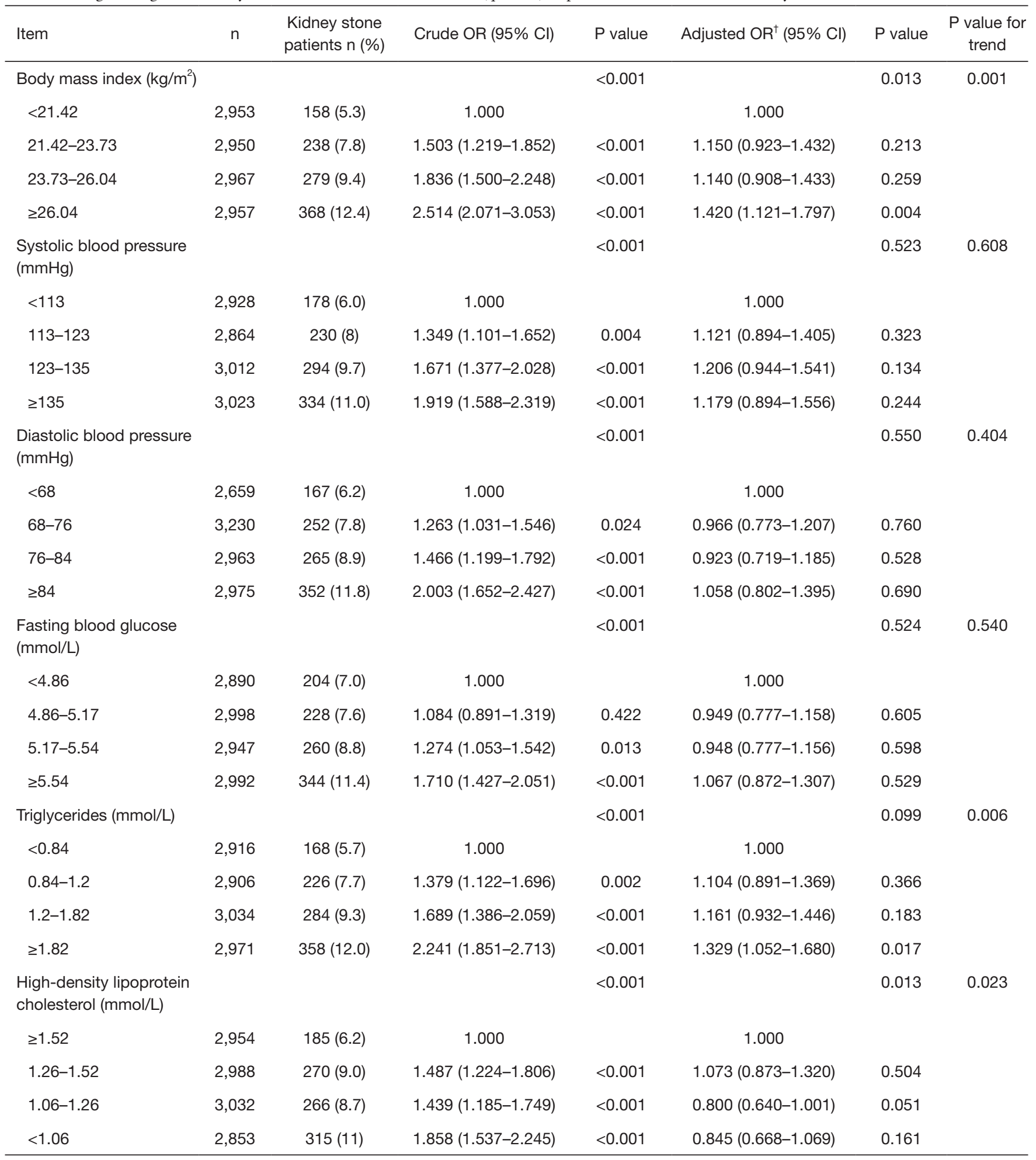

${ }^{\dagger}$, confounders were adjusted for age and sex. $\mathrm{Cl}$, confidence interval; OR, odds ratio; MetS, metabolic syndrome. 
hemoglobin) levels were significantly associated with a decrease in eGFR within 3 years (16). A cohort study of Chinese centenarians showed that age, smoking, waist circumference, SBP, and TG levels were inversely associated with glomerular filtration rate, while DBP was positively associated with glomerular filtration rate (17).

According to Table 3, we found that kidney stone morbidity in the MetS group was higher than that in the non-MetS group, and that an increase in the number of MetS characteristics was associated with an increase in kidney stone morbidity. As shown in Table 4, MetS was found to be an independent risk factor for kidney stones, with a 1.493 times higher risk than the non-MetS group (95\% CI: 1.264-1.763). Table 5 further confirmed that there was a linear trend between the number of MetS features and kidney stones. With the increase in the number of features, the risk of kidney stones also increased. Wong et al. showed that kidney stones morbidity increased with an increase in MetS features; patients with $>3$ features had higher kidney stone morbidity (12).

Logistic regression analysis was performed for specific features of MetS, and the independent variables were respectively converted into dichotomic variables according to CDS 2004 standards, or into multicategorical variables according to the interquartile interval. Results showed that BMI $\geq 25$ and blood pressure $\geq 140 / 90 \mathrm{mmHg}$ in MetS (CDS 2004) were independent risk factors for kidney stones. There was a linear trend between BMI, HDL-C, and TG in the interquartile data and dependent variables, and BMI and HDL-C were independent risk factors for kidney stone formation. Kim et al. also reported that hypertension was significantly associated with MetS (18), while Cho et al. reported that high TG were the most significant feature associated with kidney stones (15). Obese patients have other special risk factors for kidney stones compared with patients who are of normal weight, these risk factors may act through a variety of mechanisms. Given this increased risk, obese patients need more thorough and timely screening for kidney stones (19). In a mouse study, Sasaki et al. found that that weight loss intervention seemed to reduce the risk of formation of uric acid stones by improving low urine $\mathrm{pH}$ value, and reduced the risk of formation of calcium oxalate stones by increasing urinary citrate excretion (20). In terms of prevention, appropriate medication and dietary recommendations may help to correct urinary metabolic abnormalities and prevent recurrence in patients with kidney stones.

\section{Conclusions}

Most of the clinical indicators in the MetS group were statistically different from those in the control group. Abnormal weight is the primary feature of MetS in the Chinese population. Kidney stone morbidity in the MetS group was higher than that in the control group, and there was a linear trend between the number of MetS features and kidney stones. With the increase in the number of features, the risk of kidney stones also increased. MetS is an independent risk factor for kidney stones. Both BMI $\geq 25$ and blood pressure $\geq 140 / 90 \mathrm{mmHg}$ in MetS are independent risk factors for kidney stones. Appropriate medication and dietary advice may help correct urinary metabolic abnormalities and prevent kidney stone recurrence.

The present study was a cross-sectional study, which limited causal reasoning. Although the sample size was large, the composition data of kidney stones could not be obtained. Because it was based on screening data of physical examination, other components of urine, such as citrate, oxalate, phosphate, and calcium ions, cannot be collected. Therefore, the relationship between MetS and specific stone types, and the mechanism of stone formation, could not be analyzed. We can do some prospective studies in the future to further clarify.

\section{Acknowledgments}

Funding: Suzhou Science Funds for Young Scholar: KJXW2019007; Science and Technology Planning Project of Suzhou City: SLT201905.

\section{Footnote}

Reporting Checklist: The authors have completed the STROBE reporting checklist. Available at https://dx.doi. org/10.21037/tau-21-689

Data Sharing Statement: Available at https://dx.doi. org/10.21037/tau-21-689

Conflicts of Interest: All authors have completed the ICMJE uniform disclosure form (available at https://dx.doi. org/10.21037/tau-21-689). The authors have no conflicts of interest to declare.

Ethical Statement: The authors are accountable for all 
aspects of the work in ensuring that questions related to the accuracy or integrity of any part of the work are appropriately investigated and resolved. The study was approved by the Ethics Committee of First Affiliated Hospital of Soochow University (No. 178, 2021) and written informed consent was obtained from all patients. All procedures performed in this study involving human participants were in accordance with the Declaration of Helsinki (as revised in 2013).

Open Access Statement: This is an Open Access article distributed in accordance with the Creative Commons Attribution-NonCommercial-NoDerivs 4.0 International License (CC BY-NC-ND 4.0), which permits the noncommercial replication and distribution of the article with the strict proviso that no changes or edits are made and the original work is properly cited (including links to both the formal publication through the relevant DOI and the license). See: https://creativecommons.org/licenses/by-nc-nd/4.0/.

\section{References}

1. Zimmet P, Magliano D, Matsuzawa Y, et al. The metabolic syndrome: a global public health problem and a new definition. J Atheroscler Thromb 2005;12:295-300.

2. Kendall DM, Harmel AP. The metabolic syndrome, type 2 diabetes, and cardiovascular disease: understanding the role of insulin resistance. Am J Manag Care 2002;8:S63553; quiz S654-7.

3. Alberti KG, Zimmet P, Shaw J, et al. The metabolic syndrome--a new worldwide definition. Lancet 2005;366:1059-62.

4. Feng G, Feng L, Zhao Y. Association between ratio of $\gamma$-glutamyl transpeptidase to high-density lipoprotein cholesterol and prevalence of nonalcoholic fatty liver disease and metabolic syndrome: a cross-sectional study. Ann Transl Med 2020;8:634.

5. Hammarsten J, Peeker R. Urological aspects of the metabolic syndrome. Nat Rev Urol 2011;8:483-94.

6. Fernandez AM, Sherer BA, Gansky SA, et al. Ectopic biomineralization in kidney stone formers compared to non-stone formers. Transl Androl Urol 2020;9:2129-37.

7. Akman T, Binbay M, Erbin A, et al. The impact of metabolic syndrome on long-term outcomes of percutaneous nephrolithotomy (PCNL). BJU Int 2012;110:E1079-83.

8. Tiselius HG. Urinary tract stone disease: are all problems solved? Scand J Urol 2013;47:4-9.

9. Jeong IG, Kang T, Bang JK, et al. Association between metabolic syndrome and the presence of kidney stones in a screened population. Am J Kidney Dis 2011;58:383-8.

10. Ford ES, Mokdad AH, Giles WH. Trends in waist circumference among U.S. adults. Obes Res 2003;11:1223-31.

11. Tasca A. Metabolic syndrome and bariatric surgery in stone disease etiology. Curr Opin Urol 2011;21:129-33.

12. Wong Y, Cook P, Roderick P, et al. Metabolic Syndrome and Kidney Stone Disease: A Systematic Review of Literature. J Endourol 2016;30:246-53.

13. Vanhaecke T, Dolci A, Fulgoni VL 3rd, et al. Associations between urinary hydration markers and metabolic dysfunction: a cross-sectional analysis of NHANES data, 2008-2010. Eur J Nutr 2021. [Epub ahead of print]. doi: 10.1007/s00394-021-02575-3.

14. Sakhaee K, Capolongo G, Maalouf NM, et al. Metabolic syndrome and the risk of calcium stones. Nephrol Dial Transplant 2012;27:3201-9.

15. Cho ST, Jung SI, Myung SC, et al. Correlation of metabolic syndrome with urinary stone composition. Int J Urol 2013;20:208-13.

16. Kawamoto R, Akase T, Ninomiya D, et al. Metabolic syndrome is a predictor of decreased renal function among community-dwelling middle-aged and elderly Japanese. Int Urol Nephrol 2019;51:2285-94.

17. Fu S, Yao Y, Luan F, et al. Prevalence of metabolic syndrome risk factors and their relationships with renal function in Chinese centenarians. Sci Rep 2018;8:9863.

18. Kim YJ, Kim CH, Sung EJ, et al. Association of nephrolithiasis with metabolic syndrome and its components. Metabolism 2013;62:808-13.

19. Wollin DA, Skolarikos A, Preminger GM. Obesity and metabolic stone disease. Curr Opin Urol 2017;27:422-7.

20. Sasaki Y, Kohjimoto Y, Iba A, et al. Weight loss intervention reduces the risk of kidney stone formation in a rat model of metabolic syndrome. Int J Urol 2015;22:404-9.

(English Language Editor: R. Scott)

Cite this article as: Qiu F, Xu Y, Ji X, Pu J, Zhou J, Huang Y. Incidence and correlation of metabolic syndrome and kidney stones in a healthy screening population. Transl Androl Urol 2021;10(9):3646-3655. doi: 10.21037/tau-21-689 